\title{
Interacción lúdica: hacia la educación en medios. Revisión sistemática de literatura científica
}

\section{Playful interaction: towards media education. Systematic review of scientific literature}

\author{
Ángel Torres-Toukoumidis \\ Universidad Politécnica Salesiana \\ Isidro Marín-Gutiérrez \\ Universidad de Sevilla \\ Andrea De-Santis \\ Universidad Politécnica Salesiana, Universitat Autònoma de Barcelona
}

\begin{abstract}
Resumen
Internet es un espacio de aprendizaje que fomenta nuevas prácticas educativas en las cuales el uso de los medios de comunicación se vuelve una forma de alfabetización. Los alumnos del siglo XXI han crecido con Internet, con nuevos medios y con juegos, lo que ha revolucionado la forma con la cual interactúan y aprenden. Reconociendo a los juegos serios, la simulación y la gamificación como las principales tendencias aplicadas en el ámbito de la educación, este artículo desarrolla su implicación teórica en la alfabetización mediática, evidenciando cómo la mediatización digital está incluyendo juegos en su ecosistema de enseñanza-aprendizaje. Mediante un meta-análisis fundamentado en la revisión sistemática de la literatura científica, se concluye que la incorporación de las experiencias lúdicas en la alfabetización mediática se genera principalmente en la educación formal donde se logra promover actividades colaborativas que repercuten en el desarrollo creativo de los estudiantes.
\end{abstract}

Palabras clave: Alfabetización mediática; Interacción lúdica; Juegos serios; Gamificación; Simulación
ঞ

\begin{abstract}
The Internet is a learning space that encourages new educational practices in which the use of media becomes a form of literacy. Learners in the 21st century have grown up with the Internet, new media and games which have revolutionized the way they interact and learn. Recognizing serious games, simulation and gamification as the main trends applied in the field of education, this article develops its theoretical involvement in media literacy, showing how digital mediatization is including games in its teaching-learning ecosystem. Through a meta-analysis based on the systematic review of scientific literature, it is concluded that the incorporation of playful experiences in media literacy is generated mainly in formal education where it is possible to promote collaborative activities which have impact on the creative development of students.
\end{abstract}

Keywords: Media literacy; Playful interaction; Serious games; Gamification; Simulation 


\section{INTRODUCCIÓN}

Nuestra socialización viene de nuestro entorno y el de la juventud está en los medios y en los videojuegos. Internet es un espacio de aprendizaje a través de nuevas formas de comunicación y nuevas prácticas (De Santis-Piras y Jara Cobos, 2020). La comunicación mediática se convierte en herramienta y forma de alfabetización. El objetivo de este artículo es analizar la alfabetización mediática y su relación con los procesos lúdicos principalmente en la juventud. Este proceso de alfabetización se da en contextos no formales de forma colaborativa principalmente.

Podemos definir la alfabetización mediática como el conjunto de conocimientos, habilidades y competencias que una persona necesita para usar e interpretar los medios (Buckingham, 2004). Desde una visión tradicional, la alfabetización mediática tenía que ver con las capacidades de leer, ver, escuchar y entender los medios de comunicación (prensa, radio y televisión principalmente). Pero la nueva educación mediática es una comprensión crítica y de participación activa en los medios (Buckingham, 2004). Esta acción novedosa fue adoptada por la comunidad educativa actualizando los currículos escolares a partir de conceptos como las multialfabetizaciones (Gee, 2012) o las nuevas alfabetizaciones (Lacasa, 2010). Se desarrollaron nuevas teorías, como la competencia mediática (Ferrés y Piscitelli, 2012), que tratan de dimensionarla y la UNESCO propuso un currículo para desarrollar la alfabetización mediática (Wilson et al., 2013), que buscaba una educación mediática en el mundo. Otras teorías desarrollan más el llamado "alfabetismo transmedia", consistente en la convergencia mediática y el intercambio participativo de aprendizaje informal (Scolari, 2018).

Debemos encontrar una forma para que la educación mediática sea capaz de fomentar prácticas y discursos propios en el contexto de la juventud. Otro modelo es el aprendizaje conectado en comunidad tanto en entornos reales como virtuales (Ito et al., 2013). Esta cultura participativa está influyendo en los medios. La juventud actual se caracteriza por interactuar con los medios en una cultura participativa (Jenkins, 2009).

El discurso transmedia relaciona diferentes mundos narrativos en una experiencia unificada a través de los medios, en donde todos participamos (Suárez, 2019). Las audiencias se convierten en usuarios y el consumo en participación. Se crean comunidades y se comparten significados, convertidos los medios en objetos culturales más que en herramientas tecnológicas. 


\section{ALFABETIZACIÓN MEDIÁTICA Y LA INTERACCIÓN LÚDICA}

La gamificación puede beneficiar el compromiso, la motivación y el pensamiento crítico de los estudiantes y la jugabilidad no es ajena a este tipo de generación. El $97 \%$ de los adolescentes juegan a algún tipo de videojuegos. Los videojuegos proporcionan un entorno que ofrece objetivos claros, desafíos interesantes y comentarios instantáneos. Los alumnos del siglo XXI han crecido con Internet, con juegos y con nuevos medios, lo que ha revolucionado la forma con la cual interactúan con el contenido y aprenden ese contenido (Kingsley y Grabner-Hagen, 2018).

La experiencia lúdica puede ser la clave para aprovechar el poder del juego con fines educativos: de hecho, los estudiantes trabajan o juegan a actividades para aprender como si estuvieran en un museo (Men et al., 2019). Esto no significa que todas las actividades sean simplemente diversión y juegos. Los proyectos lúdicos fomentan habilidades, como la alfabetización multimodal, la alfabetización crítica, la alfabetización digital, la alfabetización mediática, la alfabetización visual, la alfabetización informativa y la alfabetización de juegos, además de las habilidades de comunicación interpersonal y el aprendizaje experimental (Gambarato y Dabagian, 2016; Marín-Gutiérrez et al., 2018).

En los juegos dentro del entorno educativo, instructores, docentes o maestros alteran una actividad o enseñan un concepto al incluir principios y mecánicas lúdicas, generalmente relacionados con la experiencia y el compromiso del estudiante, como insignias de logro, niveles y puntos de experiencia para dominar un nivel dado. El aprendizaje no está vinculado a ningún juego específico. El juego puede ser multifacético y permite la instrucción transversal. Dado el compromiso que los estudiantes sienten durante el juego es sensato incluir elementos del diseño del juego que estimulen su motivación a aprender, aún más considerando el ecosistema multiplataforma de dispositivos móviles que caracteriza su cotidianidad (De Santis-Piras y Morales Morante, 2019). Los estudiantes obtienen más puntajes después de usar los medios educativos (Wardani et al. 2019).

En definitiva, reconociendo que los juegos serios, la simulación y la gamificación son las principales tendencias aplicadas en el ámbito de la educación (Torres-Toukoumidis y Romero-Rodríguez, 2019), esta investigación pretende desarrollar la implicación teórica existente de estas tres aristas en la alfabetización mediática. En otras palabras, ¿qué relación existe entre la experiencia lúdica y el desarrollo de competencias en medios? La propuesta articulada de Ferrés y Piscitelli (2012) expresa ciertamente la eficacia de los juegos en el desarrollo de habilidades multipantalla construidas en conexión con la realidad de los medios tradicionales 
y digitales. Sin embargo, ocho años después de la publicación de tal premisa, la misma busca ser constatada en cuanto a la incursión y progresión de la experiencia lúdica. Subdivide en juegos serios, simulación y gamificación, se explora esta experiencia respecto a su configuración y a los aportes sobre a la alfabetización mediática emitidos por parte de la comunidad científica. En otras palabras, este artículo pretende explorar en qué medida la educación en medios está tomando en cuenta las didácticas lúdicas en su evolución teórica y práctica.

La investigación se ordena según el siguiente objetivo general: conducir un meta-análisis fundamentado en la revisión de literatura científica expuesta entre la alfabetización mediática y los juegos. Para ello, se establecieron dos ejes sobre los cuales se organizaron los objetivos específicos: [1] describir el contexto educativo de la alfabetización mediática que sostiene el uso de la experiencia lúdica; y [2] identificar los componentes dispuestos por la concepción lúdica en la educomunicación.

\section{Metodología}

En este estudio se utiliza la revisión sistemática de literatura científica, conocida en el entorno anglosajón como Systematic Literature Review-SLR y definida como un método riguroso que se utiliza para descubrir, contrastar y sintetizar hallazgos con la finalidad de facilitar la aprehensión de patrones y optimizar la toma de decisiones sobre procesos y fenómenos en concreto (Gough et al. 2017). Este tipo de diseño se ha aplicado continuamente en la relación educación-juegos: Torres-Toukoumidis et al. (2016) han extrapolado su aplicación para valorar las habilidades de lectura dispuestas por los videojuegos; Hainey et al. (2016) analizan la evidencia empírica del aprendizaje basado en juegos en la educación primaria; y Vlachopoulos y Makri (2017) determinan el efecto de los juegos y las simulaciones en la educación superior. En estos tres estudios, al igual que en otros (Diegmann et al., 2015; Petri y Von Wangenheim, 2016; Subhash y Cudney, 2018), se exhibe la existencia de investigaciones donde se incorpora la revisión de literatura sistemátíca entre educación y juegos.

En este sentido y aceptando la diversidad de experiencias lúdicas admitidas en la educación, el estudio se concentra únicamente en juegos serios, gamificación y simulación. Esto se debe, tal como señalan Torres-Toukoumidis y Romero-Rodríguez (2019), a su papel protagónico en el entorno educativo transmedia y su mayor impacto en los participantes en términos de motivación, compromiso, interés e incremento en las calificaciones. 
Si bien se trata de temáticas con un alto número de publicaciones cuando son analizadas de forma separada (ver Tabla 1), no existe evidencia de estudios previos que asuman este tipo de revisión sistemática entre la educomunicación y los diferentes tipos de juegos, de allí el carácter innovativo de nuestra pesquisa.

Tabla 1. Documentos en inglés y español en revistas Scopus (2009-2019) que relacionan educomunicación y tipos de juegos (elaboración propia)

\begin{tabular}{lcc}
\hline \multirow{2}{*}{ Términos utilizados en la pesquisa } & \multicolumn{2}{c}{ No de documentos $^{\circ}$} \\
& En español & En inglés \\
\hline Alfabetización mediática - Media literacy & 32 & 2.137 \\
Educación en medios & 18 & - \\
Educomunicación - Media education & 9 & 931 \\
Gamificación - Gamification & 62 & 6.174 \\
Juegos serios - Serious games & 15 & 7.482 \\
Simulación - Simulation & 841 & 2.218 .370 \\
\hline
\end{tabular}

Fuente: autores

En función de tales cuestiones, se proponen dos fases para la revisión de literatura científica: la primera fase está formalizada con los criterios de selección y diagrama de flujo expuestos por Moher et al. (2009), basado en identificación, duplicados, cribado, y sesgo; la segunda fase está precisada por los criterios análisis (ver Tabla 2 para los criterios básicos de selección y Tabla 3 para las palabras de búsqueda).

Tabla 2. Criterios básicos de selección de documentos

\begin{tabular}{ll}
\hline \multicolumn{1}{c}{ Periodo: } & \multicolumn{1}{c}{10 años: 2009-2019 } \\
\hline & Scopus \\
& Web of Science: \\
Bases de datos: & Social Science Citation Index \\
& Science Citation Index \\
& Art and Humanities Citation Index \\
\hline Idiomas: & Inglés y español \\
\hline \multirow{2}{*}{ Opciones de búsqueda: } & En Scopus: Title-Abstract-Keywords \\
& En Web of Science: Topic. \\
\hline Tipo de publicación: & Capítulos de libros \\
& Artículos \\
& Actas de conferencias \\
\hline
\end{tabular}

Fuente: autores 
Tabla 3. Palabras de búsqueda

\begin{tabular}{llll}
\hline En español & & En inglés & \\
\hline \multirow{2}{*}{$\begin{array}{l}\text { «educomunica- } \\
\text { ción» }\end{array}$} & AND «gamificación» & AND «gamification» \\
& AND «simulación» & «media literacy» & AND «simulation» \\
& AND «juegos serios» & AND «serious games» \\
\hline & AND «gamificación» & & AND «gamification» \\
$\begin{array}{l}\text { «alfabetización } \\
\text { mediática» }\end{array}$ & AND «simulación» & $\begin{array}{l}\text { «media educa- } \\
\text { tion» }\end{array}$ & AND «simulation» \\
& AND «juegos serios» & & AND «serious games» \\
\hline $\begin{array}{l}\text { «educomunicación } \\
\text { medios» }\end{array}$ & AND «gamificación» & \\
\hline & AND «simulación» & & \\
\hline
\end{tabular}

Fuente: autores

Según los criterios de selección mencionados, se presentó el diagrama de flujo (Figura 1) que resume el número de artículos extraídos para su revisión.

Figura 1. Diagrama de flujo de la investigación sobre juego y alfabetización mediática (adaptado de Moher et al. 2009).

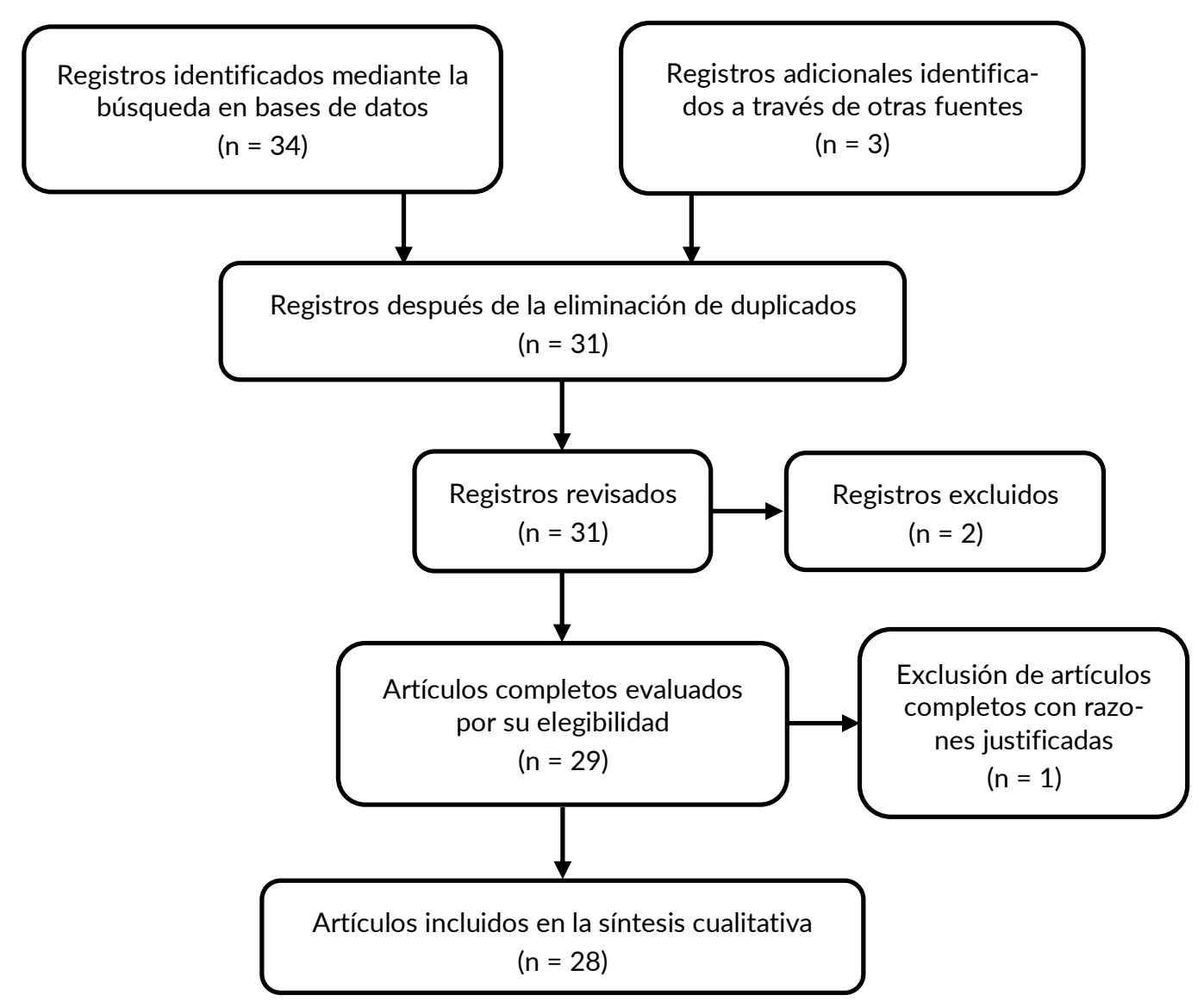


En el diagrama de flujo se evidencian 3 cribados. El primero retiró 3 documentos duplicados en la muestra (Friess et al., 2015; Huang, 2016; Katsaounidou et al., 2019). Posteriormente, en el segundo filtrado se eliminaron 2 documentos de la sección de simulación (Dumic et al. 2014; Dalyot et al., 2019) debido a que ambos se construyen hacia la modelización de estructuras de ingeniería informática y de telecomunicaciones alejando su propósito del estudio presentado. La tercera y última criba excluyó 1 documento (Lignan, 2018), a pesar de que respondía a la temática del estudio, debido a problemas de acceso al documento. En suma, el resultado de la aplicación de los criterios de selección y diagrama de flujo presenta un total de 28 documentos organizados de la siguiente manera expuesta en la figura 2.

Figura 2. Distribución de los 28 documentos seleccionados

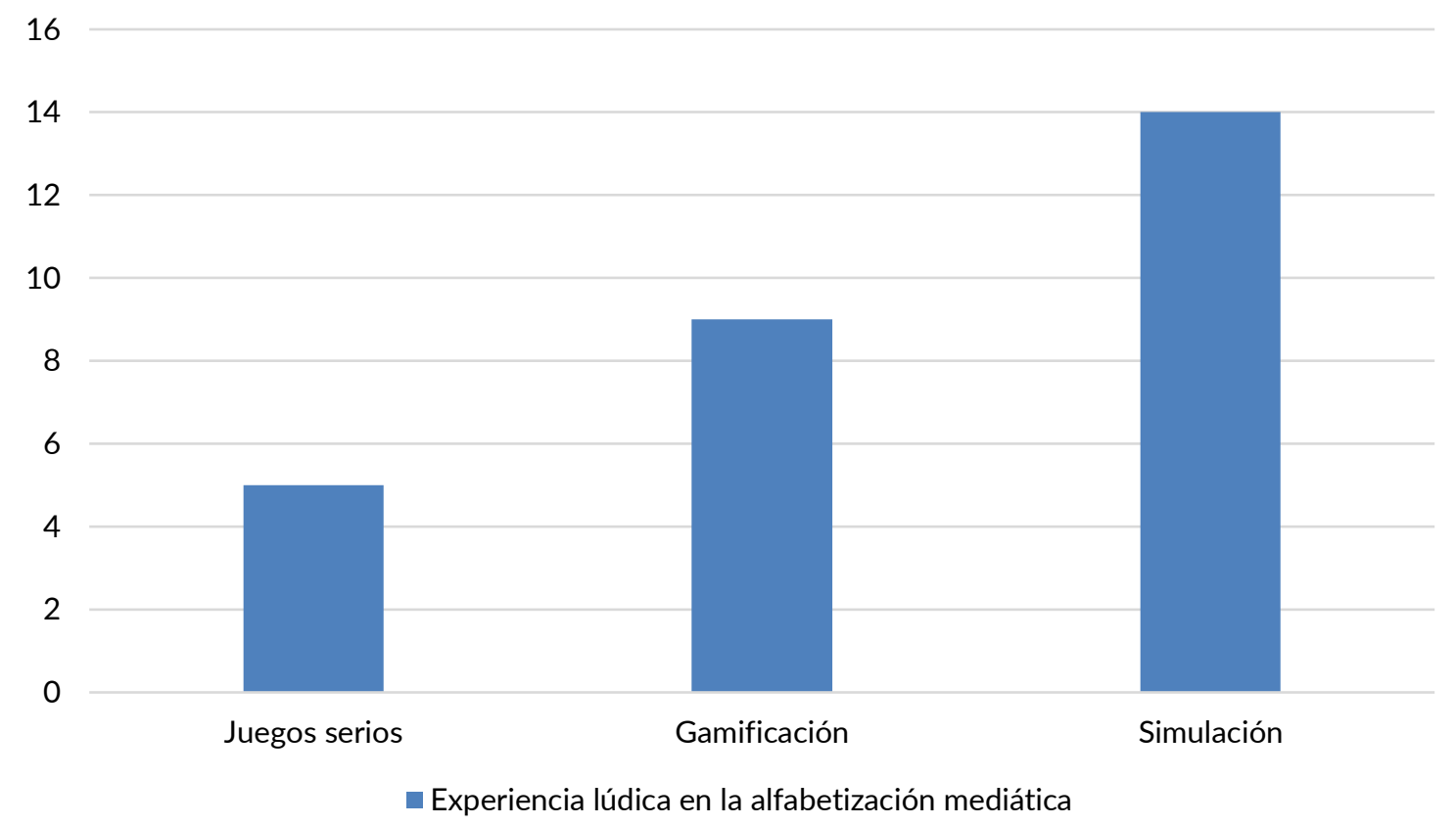

Respecto a la segunda fase, los 28 documentos seleccionados han sido revisados según 5 criterios de análisis que abordan aspectos generales, lúdicos y relacionados con la alfabetización mediática: 1) tipo de educación, 2) etapa educativa, 3 ) incorporación de dimensiones de la alfabetización mediática, 4) efecto de la experiencia lúdica, y 5) nivel de interacción.

1. Tipo de educación: formal, no formal e informal. Según Nygren et al. (2019) la educación formal es obligatoria, contiene una estructura jerarquizada y depende de un currículo académico con asignaturas y sílabo, mientras que la educación no formal se planifica fuera del sistema escolar, se construye 
con horario y programa de estudio ajustables e implica principalmente el desarrollo de habilidades profesionales; por su parte, la educación informal se refiere al proceso de enseñanza-aprendizaje natural obtenido de cualquier fuente como medios de comunicación, experiencia de vida, etc.

2. Etapa educativa. Según la clasificación de la UNESCO (2011) se organiza en 8 niveles: enseñanza preescolar, primaria, secundaria inferior, secundaria superior, postsecundaria no superior, ciclo corto de enseñanza superior, licenciatura, maestría y doctorado.

3. Incorporación de dimensiones de la alfabetización mediática. Si bien existen múltiples taxonomías para ordenar las dimensiones de este ámbito, para este estudio se ha aplicado la más citada por la comunidad académica; Ferrés y Piscitelli (2012) consideran las siguientes dimensiones:

- Lenguaje: desarrollo de la expresión del mensaje y su argumentación.

- Procesos de producción y difusión: particularización de la difusión implementada por la industria mediática.

- Proceso de interacción: relación intercultural orientada a las acciones y situaciones expuestas por el entorno.

- Ideología y valores: construcción de la visión crítica hacia las ideas que transmiten los medios.

- Estética: creatividad, innovación y sensibilidad incorporada al diseño gráfico y audiovisual de las producciones.

- Tecnología: impacto de las Tecnologías de la Información y Comunicación en el desarrollo multimedia.

4. Efecto de la experiencia lúdica. Se valora el impacto del juego en la motivación, socialización y eficacia. Li y Luh (2017) manifiestan que la motivación en la experiencia lúdica se orienta a voluntad promovida por la diversión e interés de los participantes a seguir jugando; en cuanto a la noción de socialización se configura hacia un sistema de retroalimentación teniendo como premisa la colaboración o competición entre los participantes (Lin et al., 2019); por último, la eficacia se construye hacia el desarrollo de estrategias dispuestas a proveer solución de problemas y cumplimiento de objetivos (Ong-Flaherty et al., 2017). 
5. Nivel de interacción. Dentro de la modalidad online se prescriben 3 tipos de interacción: estudiante-contenido, estudiante-docente y estudiante-estudiante; esta taxonomía se ha aplicado en el contexto lúdico orientado al aprendizaje (Cho y Cho, 2018).

Los 5 criterios mencionados ut supra proponen el descubrimiento de patrones sobre la tendencia de la vinculación entre la experiencia lúdica y la educomunicación demostrando si, en efecto, la mediatización digital está incluyendo juegos en su ecosistema de enseñanza-aprendizaje y cómo lo hace.

\section{TIPO Y NIVEL DE EDUCACIÓN DE LA ALFABETIZACIÓN MEDIÁTICA Y JUEGOS}

En cuanto al tipo de enseñanza, 21 de los documentos analizados (75\%) se han orientado al contexto formal, mientras que 4 de ellos $(14 \%)$ a la enseñanza no formal y $3(11 \%)$ a la enseñanza informal. En detalle, los documentos referidos a la enseñanza formal se ordenan de la forma señalada en la tabla 4.

Tabla 4. Documentos referidos a la educación formal por nivel educativo

\begin{tabular}{lll}
\hline \multirow{2}{*}{$\begin{array}{l}\text { Nivel educativo } \\
\text { Primaria }\end{array}$} & \multicolumn{2}{l}{$\begin{array}{l}\text { Documentos } \\
\text { No }\end{array}$} \\
$\begin{array}{l}\text { Ciclo corto de enseñanza } \\
\text { superior }\end{array}$ & 7 & $\begin{array}{l}\text { Wang et al. (2014), Friess et al. (2015), Costa et al. (2016), Huang (2016), } \\
\text { Dehart (2017), Wardani et al. (2019) } \\
\text { Leu et al. (2015), Gambarato y Dabagian (2016), Kingsley y Grabner-Hagen } \\
\text { (2018), Stoddard y Chen (2018), Göbl et al. (2019), Errabo et al. (2019), De } \\
\text { Jans et al. (2019) }\end{array}$ \\
$\begin{array}{l}\text { Licenciatura } \\
\text { Maestría }\end{array}$ & 4 & $\begin{array}{l}\text { Marín et al. (2015), Thong, et al. (2016), Huang y Yeh (2017), Katzlinger y } \\
\text { Noctorado } \\
\text { No especificador (2018) }\end{array}$ \\
\hline
\end{tabular}

Fuente: autores

El aprendizaje formal contiene la mayor proporción de publicaciones sobre educomunicación y experiencia lúdica, esto implicaría que el espacio propugnado por la enseñanza obligatoria ha tendido a vislumbrar esta combinación para el cumplimiento de los múltiples propósitos educativos. Al margen del aprendizaje formal surge el denominado no formal, que corresponde al aprendizaje que puede generarse en el entorno laboral e incluso mediante cursos de formación, dispuesto por 4 documentos, de los cuales 3 corresponden a jóvenes en general, provenientes 
de secundaria y postsecundaria (DiFranzo et al., 2019; Stoddard et al., 2017; Lukinbeal y Craine, 2009), mientras que el otro documento se orienta a profesionales de aplicaciones industriales (Quandt et al., 2018). De ello se deriva que la instrucción complementaria está igualmente condicionada por el uso de la experiencia lúdica. Para culminar, la educación informal, generada de manera natural, se evidencia hacia 3 grupos particulares: niños (Men et al., 2018), jóvenes (Živić y Zadravec, 2016) y familia, maestros, estudiantes y otros interesados (Wilson, 2012). La principal diferencia con los otros 2 tipos de enseñanza se fundamenta en la construcción de la enseñanza sin mantener una estructura curricular. De hecho, en estos casos se demuestra el uso de la experiencia lúdica en la enseñanza dentro de museos, aplicaciones móviles y reflexiones sobre la prospectiva de la educación mediática.

\section{DIMENSIONES DE LA ALFABETIZACIÓN MEDIÁTICA QUE UTILIZA LA EXPERIENCIA LÚDICA}

Admitiendo que a priori se han utilizado 6 criterios de análisis que organizan la alfabetización mediática, los hallazgos conllevan a demostrar que existe cierta aproximación entre los 13 documentos (46\%) que abordan múltiples dimensiones y aquellos que aspiran a profundizar sobre una dimensión en concreto, representados por 15 publicaciones (52\%). En la figura 3 se exhibe la puntualización hacia cada una de las dimensiones.

Figura 3. Dimensiones de la alfabetización mediática en la experiencia lúdica

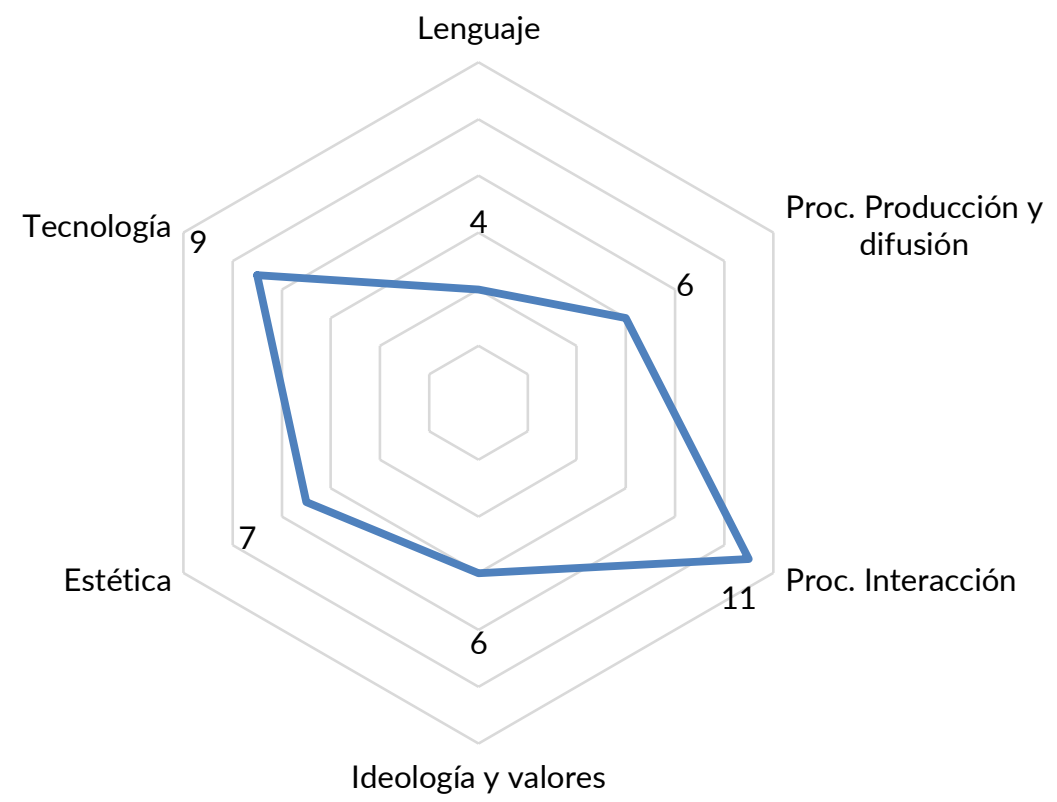


Según los datos recolectados, la dimensión denominada "Proceso de interacción" se denota en el 39\% de los documentos revisados, en referencia a la importancia de la fomentación de espacios de diálogo y acciones dispuestas por el entorno dentro del contexto mediático. Bajo esta línea, se admite la relevancia contextual para el desarrollo de habilidades de aprendizaje del siglo XXI, especialmente, en la alfabetización mediática (Errabo et al., 2019), profundizando igualmente en el análisis de las herramientas utilizadas que facilitan el desarrollo e interconexión de los estudiantes con el entorno virtual (Katzlinger y Niederländer, 2018). Además de la dicotomía virtual-presencial, el proceso de interacción también ahonda en estudios comparativos sobre accesibilidad, uso de internet y formalización de competencias desde la formulación binaria rural-urbana (Leu et al., 2015).

La dimensión denominada "Tecnología” se ubica en segunda posición, con el $32 \%$ del total de manuscritos analizados, basada en la adecuación de criterios innovativos para promover una comunicación multimedia. Esta dimensión tiene como pieza clave la repercusión del uso de las Tecnologías de la Información y la Comunicación en el conocimiento. De hecho, los casos más relevantes consisten en valorar la aceptación y aprendizaje en el uso de simulaciones de soldadura (Quandt et al., 2018), medir el impacto en el rendimiento del aprendizaje en entornos controlados de modalidad en línea (Leu et al., 2015), y analizar la adquisición de conocimientos digitales en la educación superior mediante la integración de aplicaciones y juegos móviles en el respectivo escenario de aprendizaje (Katzlinger y Niederländer, 2018).

Seguidamente, la dimensión "Estética", con el 25\% de los manuscritos, señala la promoción de la creatividad junto al diseño gráfico y audiovisual de las producciones. En este caso, destacan la participación en la creación y diseño de juego (Göbl et al., 2019; Dehart, 2017), la creación de póster de gráficos, la improvisación y demostración de multimedia (Errabo et al., 2019), los experimentos de gamificación en medios de comunicación (Wardani et al. 2019) y, por supuesto, la confección de herramientas de instrucción que sirvan de apoyo entre docentes (Dehart, 2017).

Asimismo, consta en quinto lugar la dimensión llamada "Proceso de producción y difusión" con el $21 \%$ de los documentos y que establece la ejecución particularizada del proceso de enseñanza determinada por la industria mediática en la que se observan posibilidades de diseño. Este aspecto permite a los participantes tomar decisiones en la parte experimental de la elaboración de cortometrajes 
(Friess et al., 2015), promoviendo igualmente el desarrollo de competencias audiovisuales hacia la creación de contenidos (Marín et al. 2015).

Coincidiendo con el mismo porcentaje de la "Dimensión de producción y difusión", la denominada "Ideología y valores" se conduce principalmente a sistematizar una evaluación crítica hacia los medios. Bajo esta línea, se observan estudios para detectar la censura, la comercialización o las violaciones de los derechos de autor (Živić y Zadravec, 2016), para reflexionar críticamente sobre la publicidad (De Jans et al., 2018), para modificar el comportamiento de los participantes en una actitud más crítica en lo que respecta a la evaluación y el juicio de la información en línea (Katsaounidou et al., 2019), para lograr una experiencia significativa a través de la gamificación con el fin de conducir al aprendizaje autodeterminado en el pensamiento crítico (Huang y Yeh, 2017) y, por supuesto, para desarrollar las aptitudes y conocimientos necesarios para ser más críticos con las estrategias difundidas por los medios de comunicación en el ámbito político (Stoddard et al., 2017).

La dimensión con menos propensión a estudios es el "Lenguaje", el cual se encuentra representado por el $14 \%$ de los documentos. De allí se vislumbra con especial atención el estudio de las habilidades de comunicación interpersonal en el aprendizaje experimental con robots (Gambarato y Dabagian, 2016), la enseñanza de segundas lenguas dentro de un concepto ampliado de alfabetización mediática y desde una perspectiva unificada de empoderamiento y protección (Živić y Zadravec, 2016), el mejoramiento de aptitudes de la escritura creativa (Le Rossignol, 2009) y, finalmente, la importancia del formato narrativo en el desarrollo del lenguaje (Kingsley y Grabner-Hagen, 2018).

\section{EFECTO DE LA EXPERIENCIA LÚDICA Y NIVEL DE INTERACCIÓN EN LA EDUCOMUNICACIÓN}

La implicación de la experiencia lúdica para el desarrollo de la alfabetización en medios se ha organizado en motivación, eficacia y socialización. Los efectos no son excluyentes entre sí: 12 documentos (que representan el 43\% del total) prescriben la visualización de 2 o más efectos, mientras que 16 documentos se vertebran hacia 1 solo efecto de la experiencia lúdica.

En primer lugar, la eficacia se prioriza en los estudios de la educomunicación (el 71\%, representado por 20 manuscritos), esto quiere decir que la incorporación de gamificación, simulación y juegos serios responde al estudio del desempeño de los sujetos, principalmente simbolizado por el uso de calificaciones (Friess et al., 
2015; Huang y Yeh, 2017; Stoddard y Chen, 2018; Wardani et al. 2019). Seguidamente, se encuentra la motivación, con el 39\% (11 documentos). Los estudios se enfocan principalmente en causalidades de la motivación, destacando que asumir roles para emitir críticas sobre los medios se considera una actividad extremadamente motivante (Katsaounidou et al., 2019), al igual que ocurre con la idea de incluir la creatividad como recurso de enseñanza (Costa et al., 2016) y el uso de materiales interactivos en el proceso de aprehensión (De Jans et al., 2016). Por último, la socialización corresponde al trabajo colaborativo y difusión de experiencias (el 36\% de los documentos analizados), de lo que se derivan acciones como la propuesta por Men et al. (2018) a partir de un diseño donde los estudiantes expresan una retroalimentación sobre lo aprendido en el museo, y que se corresponde con el planteamiento de Fulmore (2015) sobre el aprendizaje radicado en el paradigma constructivista hacia la personalización y trabajo colectivo. Wilson (2012), por su parte, propone que documentos oficiales de los programas de estudios educativos y análisis de las necesidades de los estudiantes deben ser a priori consensuados por los docentes, facilitando recursos en línea adecuados a la respectiva profesionalización.

Para culminar, la interacción se ha subdivido en tres niveles: estudiante-contenido ( $89 \%$, con 25 documentos), estudiante-estudiante ( $29 \%$, con 8 documentos) y estudiante-docente ( $21 \%$, con 6 documentos). La relación estudiante-contenido se confirma con el uso de plataformas digitales, interfaz que aloja el contenido disponible para la realización de las actividades (Hoyos y Gomes, 2013; Wang et al. 2014; Huang, 2016; DiFranzo et al., 2019). Respecto a la relación estudiante-estudiante, puede determinarse como interacción entre pares que conlleva a una valoración de dinámicas grupales (Leu et al., 2015; Marín et al. 2015; Huang y Yeh, 2017). Finalmente, respecto a la relación estudiante-docente, es necesario dilucidar el rol del docente, quien en su mayoría actúa de guía, mentor o instructor de las propuestas educativas, manteniendo una postura poco intervencionista y desligada del paradigma conductista (Friess et al., 2015; DeHart, 2017; Kingsley y GrabnerHagen, 2018).

\section{CONCLUSIONES}

Si bien los juegos han sido parte del aprendizaje desde los inicios de la civilización (Torres-Toukoumidis et al. 2019), la presencia de la experiencia lúdica en el aprendizaje debe asumirse como un pilar asentado desde la digitalización, la cual ha logrado proveer de consistencia interna en las competencias mediáticas mediante su incursión en el desarrollo del lenguaje, la crítica, la producción y la difusión. De 
allí que reconocer su incorporación en los últimos 10 años dentro del campo de la alfabetización mediática ha permitido acentuar su correspondencia y mimetización con los distintos tipos y niveles de educación, además de su utilidad en el proceso de enseñanza.

Específicamente, en relación al primer objetivo específico ("Describir el contexto educativo de la alfabetización mediática que sostiene el uso de la experiencia lúdica”), observamos que la alfabetización mediática se sitúa principalmente en la educación formal y en los niveles de primaria y secundaria. Sin embargo, esto no excluye la existencia de posibles casos situados en otros tipos y niveles de educación y sobre los cuales deben diversificarse los estudios, respondiendo a la necesidad de sistematizar el análisis de competencias dentro de la cotidianidad y los proyectos extracurriculares.

En relación al segundo objetivo ("Identificar los componentes dispuestos por la concepción lúdica en la educomunicación”), hemos podido alcanzar aportes de cierta trascendencia para la alfabetización mediática, entre los que se pueden evidenciar patrones como que el aprendizaje colaborativo basado en la interacción entre los jóvenes se configura como uno de los elementos fundamentales de la alfabetización mediática, que compartir herramientas como las tabletas o trabajar en pequeños grupos es visto como fomento de la creatividad, y que la interacción producida con la interfaz del contenido tiene un papel relevante en el proceso de enseñanza-aprendizaje, pudiendo sustituir al docente en ciertas prácticas como la presentación de la información y la automatización de la evaluación.

Ciertamente el estudio ha contado con limitaciones. La educomunicación y gamificación son temas emergentes dentro de la educación y el estudio de juego. Aún no han sido precisados en los currículos educativos y mantienen debates teóricos dentro de la comunidad académica. Pese a ello, se invita a futuros investigadores a profundizar en aspectos como la necesidad de reforzar el efecto de la experiencia en la construcción lingüística del mensaje y de operacionalizar la revisión del contenido de las interfaces para el beneficio de los estudiantes.

\section{REFERENCIAS}

Buckingham, D. (2004). Educación en medios: alfabetización, aprendizaje y cultura contemporánea. Paidós.

Cho, M. H., y Cho, Y. (2017). Self-regulation in three types of online interaction: a scale development. Distance Education, 38(1), 70-83.

https://doi.org/10.1080/01587919.2017.1299563 
Costa, C., Tyner, K., Henriques, S., y Sousa, C. (2016). A Review of Research Questions, Theories and Methodologies for Game-Based Learning. Journal of Content, Community \& Communication, 4(2), 2-16

Dalyot, K., Sharon, A. J., Orr, D., Ben-David, Y. B., y Baram-Tsabari, A. (2019). Public Engagement with Science in Everyday Life: Perceptions of Wi-Fi Radiation Risks in Schools. Research in Science Education, 1-20.

DeHart, J. D. (2016). Simulations, Literacy, and Learning. Journal of Adolescent \& Adult Literacy, 60(4), 485-487. https://doi.org/10.1007/s11165-019-09894-w

DeJans, S., Hudders, L., Herrewijn, L., Van Geit, K., y Cauberghe, V. (2019). Serious games going beyond the Call of Duty: Impact of an advertising literacy mini-game platform on adolescents' motivational outcomes through user experiences and learning outcomes. Cyberpsychology: Journal of Psychosocial Research on Cyberspace, 13(2). https://doi.org/10.5817/cp2019-2-3

Diegmann, P., Schmidt-Kraepelin, M., Eynden, S., y Basten, D. (2015). Benefits of augmented reality in educational environments-a systematic literature review. Wirtschaftsinformatik Proceedings 2015, 103, 1542-1556. https://aisel.aisnet.org/wi2015/103

DiFranzo, D., Choi, Y.H., Purington, A., Taft, J.G., Whitlock, J. y Bazarova, N.N. (2019). Social media testDrive: Real-world social media education for the next generation. Conference on Human Factors in Computing Systems - Proceedings. DOI: https://doi.org/10.1145/3290605.3300533

Dumic, E., Grgic, S., y Frank, D. (2014). Simulating DVB-T to DVB-T2 migration opportunities in croatian TV broadcasting. 2014 22nd International Conference on Software, Telecommunications and Computer Networks (SoftCOM) (206-210). https://doi.org/10.1109/SoftCOM33440.2014

De Santis-Piras A., y Jara Cobos, V. (2020). Comunicación estratégica de la ciencia con YouTube: el papel del comunicador/científico. En A. Torres Toukumidis y A. De Santis-Piras (Eds.), YouTube y la comunicación del siglo XXI (pp. 117-36). CIESPAL ediciones.

De Santis-Piras A., y Morales Morante, L. (2019). Dispositivos móviles y las multiplataforma de interacción lúdica. En A. Torres Toukoumidis, L. Romero-Rodríguez y J. Salgado Guerrero (Eds.), Juegos y Sociedad: desde la interacción a la inmersión para el cambio social (pp. 69-78). McGraw Hill.

Errabo, D. D. R., Berdan, M. B., y Prudente, M. S. (2019). Elucidation of essential maps for 4Cs and information-media literacy. Proceedings of the 10th International Conference on EEducation, E-Business, E-Management and E-Learning (IC4E '19), 276-280. https://doi.org/10.1145/3306500.3306553

Ferrés, J., y Piscitelli, A. (2012). La competencia mediática: propuesta articulada de dimensiones e indicadores. Comunicar, 38, 75-82. https://doi.org/10.3916/C38-2011-02-08

Friess, R., Blessing, A., Winter, J., y Zöckler, M. (2015). Film Education for Primary-School Pupils: Gamification and Interactive Storytelling as an Educational Approach to Raise Awareness of Design Structures in Feature Films. In H. Schoenau-Fog, L. Bruni, S. Louchart, S. Baceviciute (Eds.), Interactive Storytelling. ICIDS 2015. Lecture Notes in Computer Science, vol 9445 (pp.780-791). Cham: Springer.

Fulmore, Y. (2015). Video games and the customization of learning: Interactive narratives as a promising design framework for crafting inclusive educational environments. GenderIT '15: Proceedings of the Third Conference on GenderIT, 32-39. https://doi.org/10.1145/2807565.2807710

Gambarato, R.R. y Dabagian, L. (2016). Transmedia dynamics in education: the case of Robot Heart Stories. Educational Media International, 53(4), pp. 229-243. https://doi.org/10.1080/09523987.2016.1254874 
Gee, J.P. (2012). Situated language and learning: A critique of traditional schooling. Abingdon: Routledge.

Göbl, B., Hristova, D., Jovicic, S., Chevron, M.-F., Slunecko, T. y Hlavacs, H. (2019). Fostering Social Media Literacy through a Participatory Mixed-Methods Approach: Discussion of Workshop Findings. 2019 IEEE 7th International Conference on Serious Games and Applications for Health (SeGAH), 1-8. https://doi.org/10.1109/SeGAH.2019.8882464

Gough, D., Oliver, S., y Thomas, J. (Eds.). (2017). An introduction to systematic reviews. Nueva York: Sage.

Hainey, T., Connolly, T. M., Boyle, E. A., Wilson, A., y Razak, A. (2016). A systematic literature review of games-based learning empirical evidence in primary education. Computers $y$ Education, 102, 202-223. https://doi.org/10.1016/j.compedu.2016.09.001

Hoyos, C., y Gomes, M. J. (2013). Videojuegos y educación: una visión panorámica de las investigaciones desarrolladas a nivel internacional. Profesorado: Revista de Currículum y Formación del Profesorado, 17(2), 479-494. https://bit.ly/30Hazzd

Huang, H. P. (2016). Inspecting Taiwan's media education for children from the teaching cases between 1999-2011. Mass Communication Research, 129, 143-193.

Huang, L.-Y., y Yeh, Y.-C. (2017). Meaningful Gamification for Journalism Students to Enhance Their Critical Thinking Skills. International Journal of Game-Based Learning, 7(2), 4762. https://doi.org/10.4018/ijgbl.2017040104

Ito, M., Gutiérrez, K., Livingstone, S., Penuel, B., Rhodes, J., Salen, K. y Watkins, S. C. (2013). Connected learning: An agenda for research and design. Digital Media and Learning Research Hub.

Jenkins, H. (2009). Confronting the challenges of participatory culture: Media education for the 21st century. The MIT Press.

Katsaounidou, A., Vrysis, L., Kotsakis, R., Dimoulas, C. y Veglis, A. (2019). MAThE the game: A serious game for education and training in news verification. Education Sciences, 9(2), 115. https://doi.org/10.3390/educsci9020155

Katzlinger, E., y Niederländer, U. (2018). Supporting Virtual Learning for Digital Literacy: First Experiences With a Mobile app and Gamification Elements. Proceedings of the eCEL 2018 17th European Conference on e-Learning, pp. 235-244.

Kingsley, T.L. y Grabner-Hagen, M.M. (2018). Vocabulary by Gamification, Reading Teacher, 71(5), pp. 545-555. https://doi.org/10.1002/trtr.1645

Lacasa, M.P. (2010). Entrevista a Henry Jenkins: Cultura participativa y nuevas alfabetizaciones. Cuadernos de Pedagogía, 398, 52-56.

Le Rossignol, K. (2009). Designing collaborative e-learning for the net generation. IADIS 2009: Proceedings of the 2009 International Conference e-learning (pp. 183-190). IADIS Press. http://hdl.handle.net/10536/DRO/DU:30020242

Leu, D. J., Forzani, E., Rhoads, C., Maykel, C., Kennedy, C., y Timbrell, N. (2015). The new literacies of online research and comprehension: Rethinking the reading achievement gap. Reading Research Quarterly, 50(1), 37-59. https://doi.org/10.1002/rrq.85

Li, E. C., y Luh, D. B. (2017). Effect of game motivation on flow experience and companionship: the online pet games as the example. Interaction Studies, 18(1), 95-115. https://doi.org/10.1075/is.18.1.05li

Lin, Y. C., Hsieh, Y. H., Hou, H. T., y Wang, S. M. (2019). Exploring students' learning and gaming performance as well as attention through a drill-based gaming experience for environmental education. Journal of Computers in Education, 6(3), 315-334.

https://doi.org/10.1007/s40692-019-00130-y 
Lukinbeal, C., y Craine, J. (2008). Geographic media literacy: an introduction. GeoJournal, 74, 175-182. https://doi.org/10.1007/s10708-008-9216-y

Marín, V., López, M., y Maldonado, G. (2015). Can Gamification Be Introduced within Primary Classes? Digital Education Review, 27, 55-68.

Marín-Gutiérrez, I., Hinojosa Becerra, M. y Ruiz San-Miguel, J. (2018). Newsgames en Ecuador. Correspondencias \& Análisis, (8), 121-145. https://doi.org/10.24265/cian.2018.n8.06

Men, Y., Chen, R., Higgett, N. y Hu, X. (2019). A study to improve education through gamification multimedia in museum. In T. Ahram (Ed.), Advances in Intelligent Systems and Computing, 795 (pp. 294-304). https://doi.org/10.1007/978-3-319-94619-1_29

Nygren, H., Nissinen, K., Hämäläinen, R., y De Wever, B. (2019). Lifelong learning: Formal, nonformal and informal learning in the context of the use of problem-solving skills in technology-rich environments. British Journal of Educational Technology, 50(4), 1759-1770. https://doi.org/10.1111/bjet.12807

Ong-Flaherty, C., Valencia-Garcia, D., Martinez, D. A., Borges, W., y Summers, L. (2017). Effectiveness of gaming in creating cultural awareness. Learning, Culture and Social Interaction, 12, 149-158. https://doi.org/10.1016/j.lcsi.2016.12.005

Petri, G., y Von Wangenheim, C. G. (2016). How to evaluate educational games: a systematic. Journal of Universal Computer Science, 22(7), 992-1021. https://bit.ly/3jlPD75

Quandt, M., Knoke, B., Gorldt, C., Freitag, M., y Thoben, K.-D. (2018). General Requirements for Industrial Augmented Reality Applications. Procedia CIRP, 72, 1130-1135. https://doi.org/10.1016/i.procir.2018.03.061

Stoddard, J., y Chen, J. (2018). Exploring the Impact of Virtual Internships for Democratic and Media Education. 13th International Conference of the Learning Sciences, ICLS, pp. 1695-1696. https://bit.ly/31NY1GO

Stoddard, J., Rodriguez, K., Rayner, M. Swiecki, Z. y Shaffer, D.W. (2017). Epistemic game design for democratic and media education. Computer-Supported Collaborative Learning Conference, CSCL, 2, 769-770. https://bit.ly/3cP2LxE

Suarez, M. F. (2019). An interview with Dr Carmen Herrero, an expert in film and language education. Bellaterra Journal of Teaching \& Learning Language \& Literature, 12(4), 113118. https://doi.org/10.5565/rev/jt13.837

Subhash, S., y Cudney, E. A. (2018). Gamified learning in higher education: A systematic review of the literature. Computers in Human Behavior, 87, 192-206. https://doi.org/10.1016/i.chb.2018.05.028

Thong, L. P., Stewart, C., Arnab, S., y Lameras, P. (2016). Virtual Designer: Digital Role-Playing Game for Knowledge Transferal in Design Education. European Conference on Games Based Learning, 862-869. Academic Conferences International Limited.

Torres-Toukoumidis, Á., y Romero-Rodríguez, L. M. (2019). Gamificación, simulación, juegos serios y aprendizaje basado en juegos. En A. Torres-Toukoumidis, L. Romero-Rodríguez y J. Salgado (Eds.), Juegos y sociedad: desde la interacción a la inmersión para el cambio social, (pp. 113-135). McGraw Hill.

Torres Toukoumidis, Á., Romero Rodríguez, L. M., Pérez Rodríguez, M. A., y Björk, S. (2016). Desarrollo de habilidades de lectura a través de los videojuegos: Estado del arte. Ocnos: Revista de estudios sobre lectura, 15(2), 37-49. https://doi.org/10.18239/ocnos_2016.15.2.1124

Unesco. (2012). International Standard Classification of Education ISCED 2011.. Montreal: UNESCO Institute for Statistics. international-standard-classification-of-education-isced2011-en.pdf (unesco.org) 
Vlachopoulos, D., y Makri, A. (2017). The effect of games and simulations on higher education: a systematic literature review. International Journal of Educational Technology in Higher Education, 14, 22. https://doi.org/10.1186/s41239-017-0062-1

Wang, Y., Zhang, Y., y Gu, L. (2014). Proxy Caching Algorithm based on Segment Group Popularity for Streaming Media. International Journal of Multimedia and Ubiquitous Engineering, 9(2), 297-308. https://doi.org/10.14257/ijmue.2014.9.2.30

Wardani, I., Tolle, H. y Aknuranda, I. (2019). Evaluation of an educational media on cube nets based on learning effectiveness and gamification parameters. International Journal of Emerging Technologies in Learning, 14(14), pp. 4-18. DOI: https://doi.org/10.3991/ijet.v14i14.10505

Wilson, C. (2012). Alfabetización mediática e informacional: proyecciones didácticas. Comunicar, 39, 15-24. https://doi.org/10.3916/c39-2012-02-01

Wilson, C., Grizzle, A., Tuazon, R., Akyempong, K., y Cheung, C.-K. (2013). Alfabetización Mediática e Informacional Curriculum para profesores. UNESCO. https://unesdoc.unesco.org/ark:/48223/pf0000216099.locale=en

Živić, T., y Zadravec, T. (2016). English Language as a Promoter of Media Literacy Education. In S. Kurbanoğlu, J. Boustany, S. Špiranec, E. Grassian, D. Mizrachi, L. Roy y T. Çakmak (Eds.), Information Literacy: Key to an Inclusive Society (pp. 147-156). Springer. https://doi.org/10.1007/978-3-319-52162-6_15

\section{ÁNGEL TORRES-TOUKOUMIDIS}

Doctor en Comunicación bajo la línea de investigación "Alfabetización de los Medios", de las universidades de Huelva, Sevilla, Málaga y Cádiz. Precursor del primer laboratorio universitario de juegos en Ecuador. Miembro del Grupo de Investigación sobre Misiones y Pueblos Indígenas (GIMPI). Actualmente es profesor en la Universidad Politécnica Salesiana, Ecuador.

atorrest@ups.edu.ec https://orcid.org/0000-0002-7727-3985

\section{ISIDRO MARÍN-GUTIÉRREZ}

Profesor Asociado del departamento de Sociología y Trabajo Social en la Universidad de Huelva. Profesor-Tutor en el Centro Asociado de la UNED de Huelva. Profesor Ayudante Doctor en la Universidad de Sevilla, España.

imarin1@utpl.edu.ec https://orcid.org/0000-0002-6858-0983

\section{ANDREA DE-SANTIS}

Doctorando en Comunicación Estratégica, Publicidad y Relaciones Publicas en la Universidad Autónoma de Barcelona, Magister en Dirección de Comunicación Empresarial e Institucional (UDLA), y Dottore in Scienze della Comunicazione (Universidad La Sapienza di Roma). Director y profesor titular del programa de Comunicación de la Universidad Politécnica Salesiana, Ecuador. Miembro del GICEA (Grupo de Investigación Comunicación, Educación y Ambiente).

adesantis@ups.edu.ec https://orcid.org/0000-0002-7441-3794 


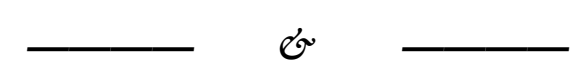

Torres-Toukoumidis, Á., Marín-Gutiérrez, I. y De-Santis, A. (2021). Interacción lúdica: hacia la educación en medios. Revisión sistemática de literatura científica. Bellaterra Journal of Teaching \& Learning Language \& Literature, 14(3), e940. https://doi.org/10.5565/rev/jt13.940

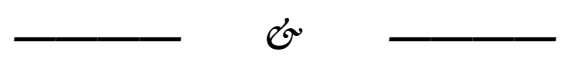

Rebut / Recibido / Received / Reçu: 20-08-2021

Acceptat / Aceptado / Accepted / Accepté: 09-09-2021 